\title{
Innate Responses to Putative Ancestral Hosts: Is the Attraction of Western Flower Thrips to Pine Pollen a Result of Relict Olfactory Receptors?
}

\author{
Zayed S. Abdullah • Katherine J. Ficken • \\ Bethany P. J. Greenfield • Tariq M. Butt
}

Received: 13 February 2014 / Revised: 9 April 2014 / Accepted: 19 May 2014 / Published online: 31 May 2014

(C) The Author(s) 2014. This article is published with open access at Springerlink.com

\begin{abstract}
Pollinophagy is widely documented in the order Thysanoptera, with representative individuals from six of the nine divergent families known to feed on pollen. Various pollens of the genus Pinus increase the development time, fecundity, longevity, and settling preference of Western Flower Thrips (WFT), Frankliniella occidentalis (Pergande) (Thysanoptera: Thripidae). Certain species of flower thrips discriminate among pollen types, but no studies have elucidated the olfactory cues that play a role in their pollen preferences. In this study, the volatile organic compounds emitted by pollens of the genus Pinus were elucidated. Various chemicals from pollen headspace elicited electrophysiological responses from WFT antennae. The compound (S)-(-)verbenone, identified in pollen headspace, attracted WFT in a 4-arm olfactometer. This compound has potential for use in integrated pest management programs against the pest. We present the hypothesis that this polyphagous insect may have retained ancestral 'relict' olfactory receptors through the course of evolution, to explain this attraction to pine pollen. This attraction has allowed the insect to find and exploit an unusual nutrient source that significantly increases its fitness. The study demonstrates how fossil record analysis and subsequent evolutionary knowledge can aid in explaining possibilities as to why some insects sense and respond to chemicals that would otherwise seem peculiar to their ecology, allowing
\end{abstract}

Electronic supplementary material The online version of this article (doi:10.1007/s10886-014-0450-0) contains supplementary material, which is available to authorized users.

Z. S. Abdullah $(\bowtie) \cdot$ B. P. J. Greenfield · T. M. Butt

Department of Biosciences, Swansea University, Singleton Park,

Swansea SA2 8PP, UK

e-mail: 489458@swansea.ac.uk

K. J. Ficken

Department of Geography, Swansea University, Singleton Park,

Swansea SA2 8PP, UK insight into the evolutionary forces that may shape insect olfactory systems over time.

Keywords Frankliniella occidentalis · Thrips · Pine pollen · Semiochemical · (S)-(-)-Verbenone · Electroantennogram · Insect pest

\section{Introduction}

Thrips (Thysanoptera) of two sub-orders, comprising six of the nine divergent families, supplement their diet with various pollens, a highly nutritious but non-essential food source (Heming 1993). Representative species from three families feed on pollen by using their forelegs to grab individual grains (Kirk 1984, 1985, 1987), a method of feeding that differs slightly from that used in feeding on plant tissue. This suggests that pollinophagy is either a primitive feature or that it has evolved independently in several families of Thysanoptera. There is fossil evidence of Mesozoic fossilized thrips carrying pollen of ancient gymnosperms (Penalver et al. 2012), consistent with pollinophagy being a primitive trait in the order.

Western Flower Thrips (WFT), Frankliniella occidentalis (Pergande) (Thysanoptera: Thripidae) is a major agricultural and horticulture pest worldwide (Kirk 2002; Kirk and Terry 2003). This insect causes damage and spoilage to a large number of economically important plant species through feeding, oviposition, and spread of several plant diseases, notably tospoviruses (Morse and Hoddle 2006). The cryptic nature and small size of this insect means that it can remain undetected through quarantine control measures. Its distribution throughout the world has been facilitated by the increase in international plant movement (Kiritani 2001). A major concern with this insect is the rapid development of insecticide resistance in populations (Bielza et al. 2007; Bielza 2008). This 
problem is expected to become more important because many pesticides have been withdrawn from use in the EU (Directive 2009/128/EC (2009)). This highlights the need to develop alternative control and monitoring methods.

Various pollens of the genus Pinus increase the development time, fecundity, longevity, and settling preference of WFT (Chitturi et al. 2006; Hulshof and Vanninen 2002). Separate studies suggest that WFT population dispersal may be positively correlated with pine pollen dispersal (Chitturi et al. 2006; Riley et al. 2007, 2011). Both pollen and the oily coating of pollen grains, known as pollenkitt, produce speciesspecific odors that can be distinguished by bees (Dobson 1987). Certain species of flower thrips have been shown to discriminate among host plant pollen (Kirk 1984, 1985), but no studies have elucidated the olfactory cues that play a role in this preference of thrips.

Our study is the first, to our knowledge, that has identified the volatile organic compounds (VOCs) emitted by pine pollen and tested what VOCs elicit both electrophysiological and behavioral responses in WFT. These chemicals could be incorporated into new integrated pest management strategies targeting WFT, as well as give us a better understanding of the olfactory capabilities of this pest.

\section{Materials and Methods}

Rearing A colony of WFT, provided by Keele University, UK, was maintained on a bouquet of mixed cultivar chrysanthemum, Dendranthema grandiflora, potted in multi-purpose growing media (to allow for pupation of thrips), and kept on capillary matting inside ventilated Perspex cages (L $30 \times \mathrm{W}$ $30 \times \mathrm{H} 60 \mathrm{~cm})$ at $25 \pm 2{ }^{\circ} \mathrm{C}$ and L18: D6 photoperiod. The capillary matting was kept damp with distilled water. A fresh bouquet was placed in the rearing chamber every week, such that three bouquets were present in the rearing chamber at any one time. The oldest bouquet was removed upon addition of a new bouquet; this cycle provided optimal rearing conditions.

Pollen Pinus elliotti pollen was collected by members of the Department of Entomology, University of Georgia, near Athens, Georgia, USA. Pinus sylvestris and Pinus massoniana pollen was purchased from Amla Berry Ltd (New Delhi, India), and NatuHealth Ltd (Dundee, UK), respectively. All pollen samples were stored at $-80{ }^{\circ} \mathrm{C}$ and used within one month of acquisition.

Volatile Collections Pollen volatiles were collected using static headspace Solid Phase Microextraction (SPME). Ten milligrams of each pine pollen sample were placed into specialized $300 \mu \mathrm{l}$ glass inserts in $2 \mathrm{ml}$ screw top vials (03-FISV, Chromacol Ltd, Herts, UK), capped with $9 \mathrm{~mm}$ silicone/polytetrafluoroethylene screw caps [9-
SC(B)-ST1, Chromacol Ltd, Herts, UK]. Pollen volatiles were allowed to equilibrate in the headspace for $1 \mathrm{~h}$ at room temperature (ca. $24{ }^{\circ} \mathrm{C}$ ) prior to collection. For collection of headspace volatiles, a 50/30 DVB/ Carboxen $^{\mathrm{TM}} /$ PDMS StableFlex ${ }^{\mathrm{TM}}$ SPME fiber (Gray Fibre, Supelco) was inserted through the septum and exposed for $1 \mathrm{~h}$. The fiber was desorbed in a gas chromatograph (GC) injection port within $5 \mathrm{~min}$ of retraction. Fibers were preconditioned as per the manufacturer's guidelines, with a blank run always performed before adsorption so as to ensure the fibers were free of contamination. Three replicates were carried out in this fashion, using three batches of each of the three pollen samples. For standards, the same procedure was used depositing $1 \mu \mathrm{l}$ of $n$-hexane containing $100 \mathrm{ng}$ of test chemical into the same vials.

Gas Chromatography/Mass Spectrometry Gas chromatography/mass spectrometry (GC/MS) analysis was carried out on an HP6890 gas chromatograph coupled to a 5975 inert Mass Selective Detector (Agilent Technologies) operated in electron impact ionization (EI) mode (at $70 \mathrm{eV}$ ). SPME fibers were inserted into the GC split/splitless injection port (at $230{ }^{\circ} \mathrm{C}$ ), fitted with a Merlin Microseal (ThamesResteck, High-Wycombe, UK), and operated in splitless mode. A 2 min desorption time was allowed. The GC was fitted with an HP-5MS ( $\mathrm{J}$ and $\mathrm{W}$ Scientific) fused silica capillary column $(30 \mathrm{~m} \times 0.25 \mathrm{~mm} \times 0.25 \mathrm{~mm}$ film thickness $)$. The oven temperature was held at $40^{\circ} \mathrm{C}$ for $2 \mathrm{~min}$ and then increased by $10{ }^{\circ} \mathrm{C} \cdot \mathrm{min}^{-1}$ to $250{ }^{\circ} \mathrm{C}$. Helium was used as carrier gas. Tentative identifications were made by comparing spectra with those of authentic samples in two separate mass spectral databases: NIST, 2011 version and Wiley 9th edition. Authentic standards, if available, were used to confirm our tentative identifications (although we did not identify specific enantiomers). Blanks were run of empty vials to eliminate background peaks. In order to determine which chemicals the thrips had been exposed to in the rearing chamber, volatile collections of the headspace of fresh chrysanthemum bouquets also were analyzed.

Chemicals The purities and sources of chemicals used in this study are listed in Table 1.

Electrophysiological Recordings of Adult Female WFT Antennae Electroantennograms (EAGs) of the antennae of WFT to pollen VOCs were carried out as follows. The anterior portion of an adult female WFT was excised at the groove at which the mesonotum connects to the pronotum, and both antennae excised at the groove between antennal segments 7 8 (most distal to the head) to allow for better contact with the electrodes. The preparation was mounted by placing the most proximal end into the reference electrode and both antennae 
Table 1 Sources and purities of authentic chemical standards

\begin{tabular}{|c|c|c|}
\hline Chemical & Source & Purity \\
\hline (-)-borneol & Sigma Aldrich & $97 \%$ \\
\hline (-)-bornyl acetate & Sigma Aldrich & $95 \%$ \\
\hline (-)-caryophyllene oxide & Sigma Aldrich & $\geq 99 \%$ \\
\hline (-)-trans-caryophyllene & Sigma Aldrich & $\geq 98.5 \%$ \\
\hline$(+)$-longifolene & MP Biomedicals & Analytical Standard \\
\hline$(1 S)-(-)-\beta$-pinene & Sigma Aldrich & $99 \%$ \\
\hline (S)-(-)-camphor & Fluka & $\geq 99 \%$ \\
\hline$(S)$-(-)-verbenone & Sigma Aldrich & $\geq 93 \%$ \\
\hline (Z)-pinocarveol & Sigma Aldrich & $\geq 96 \%$ \\
\hline$(1 R)$ - $\alpha$-pinene & Sigma Aldrich & $98 \%$ \\
\hline$(1 R)-(-)-$ myrtenal & Sigma Aldrich & $98 \%$ \\
\hline acetic acid & Fluka & $\geq 99.5 \%$ \\
\hline ethyl acetate & Fisher Scientific & HPLC Grade \\
\hline$\gamma$-butyrolactone & Sigma Aldrich & $\geq 99 \%$ \\
\hline humulene ( $\alpha$-caryophyllene) & Sigma Aldrich & $\geq 96 \%$ \\
\hline isoamyl alcohol (3-methyl-1-Butanol) & Sigma Aldrich & $\geq 98 \%$ \\
\hline (-)-terpinen-4-ol & Sigma Aldrich & $\geq 95 \%$ \\
\hline$\alpha$-terpineol & Sigma Aldrich & $\geq 96 \%$ \\
\hline (Z)-3-hexen-1-ol & Sigma Aldrich & $97 \%$ \\
\hline
\end{tabular}

into the recording electrode, with the aid of micromanipulators. The glass electrodes were filled with glucose-free Ringer's solution (Maddrell 1969). Authentic standards (in $10 \mu \mathrm{l}$ of hexane) were applied to strips of filter paper at a dose of $1 \mathrm{mg}$. A filter paper was placed in a Pasteur pipet and allowed to sit for at least a minute to allow the solvent to evaporate. The pipet was then attached to a $5 \mathrm{ml}$ syringe via rubber tubing. The syringe plunger was depressed (over less than $2 \mathrm{sec}$ ), expelling the volatile contents of the pipet into a purified airstream at a flow of $11 . \mathrm{min}^{-1}$ through a glass tube (i.d. $120 \mathrm{~mm}$ ) over the preparation. Separate syringes were used for each chemical. This method gave consistent EAG peaks using a standard of (Z)-3-hexen-1-ol (see Fig. S1a in the electronic supplementary material). The EAG equipment consisted of a $10 \times$ gain universal probe (Syntech, Netherlands) and an IDAC 2 Signal Acquisition Processor (Syntech). Data were analyzed with EAGPro Version 2 software, (Syntech). (Z)-3-Hexen-1-ol standard was tested at the start of each replicate, and these responses were used to normalize all test recordings to the largest response recorded in an experiment. Responses to a hexane control were measured at the start and end of each preparation; these were normalized and averaged. Nine recordings were made to each test compound using a minimum of three insects. Data were square root transformed for homogeneity of variance, and analyzed using a general linear model. The model incorporated fixed effects of treatment, with insect specimen used for each recording included as a random factor. Means were compared using a Dunnet's post-hoc test (comparing treatments to the hexane control) with SPSS software (IBM Corporation, USA).

Bioassays of Adult Female WFT to Pine Pollen, Chrysanthemum Bud and Chemicals A Perspex four-arm olfactometer (Pettersson 1970), with an arena diameter (arm to opposite arm) of $120 \mathrm{~mm}$ was used to determine behavioral responses of adult female WFT to whole pollen samples and to chemicals identified in pollen headspace. Samples were placed in $14 \mathrm{~mm}$ i.d. glass arms with an i.d. of $3 \mathrm{~mm}$ at the connecting point. Prior to any experiment, all Perspex components of the olfactometer were washed with Teepol detergent (SupplyTrade Ltd, Kent, UK), rinsed with $75 \%$ ethanol, followed by distilled water, and then left to dry overnight. All glassware was washed with Teepol detergent, rinsed with acetone, followed by water and heated overnight at $400{ }^{\circ} \mathrm{C}$. The olfactometer was placed in a cardboard box (20 cm high) lined with thick black plastic sheeting to exclude visual cues. The light intensity was measured at 400 lux at the top of the box using a digital light meter (Model: LX1010B, Sinometer Instruments, China). The UV intensity was measured at $0.2 \mu \mathrm{W} / \mathrm{cm}^{2}$ using a UVA meter (Tecpel 830, Tecpel Co. Ltd, Taiwan). Three of the olfactometer arms were used for controls and the fourth for a treatment. The arms contained glass wool in their tips to prevent insects escaping, as well as to block any external visual cues. A filter paper disc (Whatman $^{\mathrm{TM}} 1$ Qualitative), for traction, lined the base of the olfactometer, as well as providing good contrast by which to observe the insect. Both pollen and standard chemicals 
were bioassayed. When testing pollen, $10 \mathrm{mg}$ were placed on a $1 \mathrm{~cm}^{2}$ filter paper piece in the treatment arm, and blank filter paper pieces were placed in each control arm. For chemical standards, $10 \mu \mathrm{g}$ of chemical (in $10 \mu$ l of hexane) were applied to a $1 \mathrm{~cm}^{2}$ filter paper piece; controls consisted of $10 \mu \mathrm{l}$ of hexane applied to filter paper pieces. A positive control consisted of a chrysanthemum bud in the treatment arm and blanks in the control arms. The bioassay was started $5 \mathrm{~min}$. after introduction of the samples into the olfactometer, to allow for a steady release of VOCs.

Adult female WFT were conditioned in a Petri dish containing a small piece of wet filter paper for at least $4 \mathrm{~h}$ prior to the start of an experiment. An individual thrips was placed in the top central hole of the olfactometer using a dampened fine tip paintbrush; a piece of glass tube was fitted into this hole and sealed with a piece of Blu-Tack (Bostik, Paris, France). The glass tube was connected to a rotameter (Supelco, UK) and air drawn through the central hole at a rate of $400 \mathrm{ml} . \mathrm{min}^{-1}$ using a $12 \mathrm{~V}$ rotary vane pump (Gardner Denver Ltd, Hampshire, UK). The olfactometer was divided into four equal sections. A thrips was given $1 \mathrm{~min}$ to acclimate to the olfactometer, before the total time it spent in the treated section was recorded over $16 \mathrm{~min}$. During the experiment, the olfactometer was rotated every $4 \mathrm{~min}$. Twelve replicates were carried out per treatment. Time spent in the treated section was analyzed using SPSS (IBM corporation, USA). Time spent in the treated region was converted to a percentage (of the $16 \mathrm{~min}$.), logit transformed and compared to a test mean of -1.099 (logit transformation of $25 \%$ ) using a one sample $t$ test; $25 \%$ being the time an insect would be expected to spend in a region randomly. A significantly higher amount of time in the treated region was interpreted as an insect showing attraction to a test odor, while a statistically lower amount of time in the treated region was interpreted as an insect showing avoidance.

\section{Results}

Volatile Compounds in Headspace of Pine Pollen GC/MS analysis of the headspace of pollen from the three Pinus species (Tables S2a-c in the electronic supplementary material) showed that all three released $\alpha$-pinene, $\beta$-pinene, borneol, $\beta$-caryophyllene, bornyl acetate, and verbenone. Terpinen-4ol and $\alpha$-terpineol were found in the headspace of P. massoniana and P. elliotti, while caryophyllene oxide, longifolene, and acetic acid were found in the headspace of both $P$. sylvestris and $P$. massoniana. Ethyl acetate, isoamyl alcohol, camphor, and $\alpha$-humulene were found only in the headspace of $P$. elliotti, while pinocarveol, myrtenal, and $\gamma$ butyrolactone were detected only in the headspace of P. massoniana pollen. $\beta$-Pinene, camphor, borneol, bornyl acetate, $\beta$-caryophyllene, and caryophyllene oxide were detected in the headspace of both pine pollen and fresh chrysanthemum bouquets (Fig. S2a-d shows total ion chromatograms and overlays).

Electrophysiological Responses of WFT to Volatile Compounds from Pine Pollen Significant (different from the $n$ hexane control) EAG responses of WFT (Fig. 1) were recorded to isoamyl alcohol, ethyl acetate, (-)-borneol, $\alpha$-humulene, and $(S)-(-)$-verbenone (all $P<0.001),( \pm)-\alpha$-terpineol $(P<0.01)$, and $\gamma$-butyrolactone and caryophyllene oxide (both $P<0.05)$. All other chemicals tested showed EAG responses that were not different to that from the $n$-hexane control. For representative EAG recordings of thrips to chemicals detected in pine pollen, see Figure S1b-t.

Behavioral Responses of WFT to Pine Pollen and Individual Compounds WFT showed attraction to odors of P. massoniana $(P=<0.05)$ and $P$. elliotti pollen $(P<0.01)$, but not to $P$. sylvestris pollen (Fig. 2). Of the individual chemicals, WFT showed attraction only to $(S)$-(-)-verbenone $(P<0.01)$. WFT exhibited avoidance to isoamyl alcohol $(P<0.05)$ and $\gamma$-butyrolactone $(P<0.01)$. WFT showed attraction to odors of a chrysanthemum bud (positive control), but not to the blank $n$-hexane (Fig. 3 ).

\section{Discussion}

Pollen of $P$. elliotti, $P$ sylvestris, and $P$. massoniana had similar VOC headspace profiles, consisting mostly of terpenoids, with few compounds unique to any one species. That that pines diverged from the closely related sister genus Picea some 87193 million years ago (Grotkopp et al. 2004), and yet the three pine pollen samples had similar VOC profiles, suggests a relatively stable rate of odor evolution within the genus Pinus, at least with regards to the synthesis of pollen terpenoids. Compounds unique to specific pine species may be a result of environmental stress factors (e.g., humidity, temperature, light, and nitrogen availability), which are known to evoke variability in volatile emission profiles among plants of the same species (Gouinguené and Turlings 2002). Pollen volatiles are chemically diverse compounds that may play multiple roles, and their production and emission may have evolved under conflicting selective pressures that protect the male gametophyte but also encourage dispersal by insects (Dobson and Bergstrom 2000). Several terpenoids (e.g., caryophyllene, longifolene) detected in the headspace of pine pollen are known to have antibiotic properties and may protect against pathogens (De Moraes et al. 2001; Friedman et al. 2002; Hammer et al. 2003).

WFT are attracted to crude pine pollen (Chitturi et al. 2006), and certain flower thrips can discriminate among pollen of different plant species (Kirk 1984, 1985). Our study shows, for the first time, that specific olfactory cues mediate the attraction of WFT to pine pollen. Of the identified compounds in pine 
Fig. 1 Mean electroantennogram (EAG) responses (normalized to (Z)-3-hexen-1-ol) of adult female western flower thrips to compounds identified from three pine pollen samples $(N=9)$ (data presented as mean $\pm 95 \%$ C.I., ***- $P<0.001, * *_{-} P<0.01$, * $P<0.05$, compared to $n$-hexane control; general linear model, Dunnet's post-hoc test)

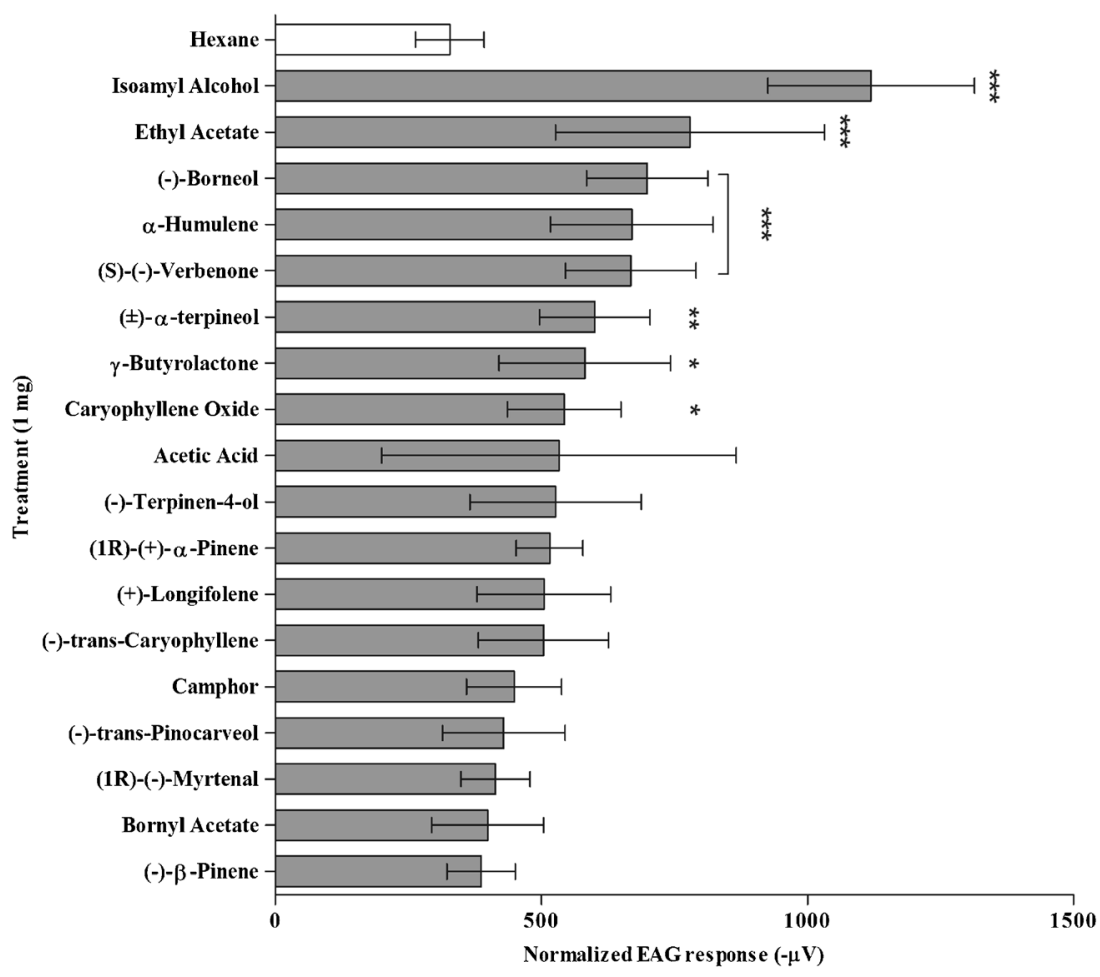

pollen, WFT showed electrophysiological responses and attraction to $(S)$-(-)-verbenone, suggesting that it is a major component responsible for attraction and perhaps preference (over other pollens) to pine pollen. This compound was released by pollen of all three Pinus species. (S)-(-)-Verbenone has not been widely reported as a semiochemical. It is known to repel certain coleopterans and attract others in coniferous forests (Paine and Hanlon 1991; Payne and Billings 1989; Progar 2005; Schlyter

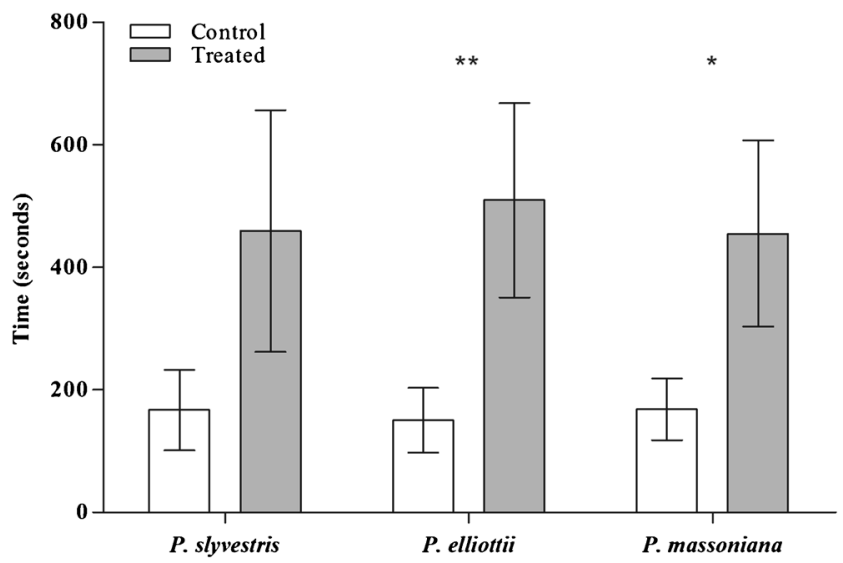

Fig. 2 Mean responses of adult female western flower thrips to pollen odors from different Pinus species in a four-arm olfactometer. Time spent in the treated section was converted to a percentage of overall time, logit transformed, and compared to a test mean of -1.099 (logit transformation of $25 \%$ ) by a one-sample $t$-test. Data presented as $\pm 95 \%$ C.I., **$\left.P<0.01, *_{-} P<0.05\right), N=12$ et al. 1989; Shea et al. 1992; Shore et al. 1992; Tilden and Bedard 1988). Headspace analysis of the chrysanthemums on which the insects were reared showed no trace of $(S)-(-)$ verbenone; hence, the response of WFT to this compound is likely innate.

Such an innate response to (S)-(-)-verbenone may have evolved in the last 40-50 million, a period during which most of the taxa that comprise the recent fauna of the order Thysanoptera evolved, presumably coinciding with the radiation of angiosperms (Grimaldi et al. 2004). Alternatively, such genetic refinement and development of a chemoreceptor may have evolved during the pre-angiosperm Mesozoic period, when gymnosperm-insect co-evolution was at its peak (Labandeira 2006), and has been retained as what could be termed a 'relict' olfactory receptor: a receptor that carries out secondary functions in extant species that were once involved with primary functions in their ancestors. We speculate that gymnosperm plants may have then been primary hosts to the ancestors of WFT. Clearly, individuals that are able to locate the most nutritious food sources, whether pollen or plant parts, would have a competitive advantage and concomitantly associated alleles would be conserved over evolution. Evidence to support such an idea could be provided by single-sensillum studies on the specificity of specific olfactory receptor neurons (ORNs) to (S)-(-)-verbenone and other structurally related compounds. In addition, studies on the presence of (S)-(-)verbenone-specific ORNs in species across many families of 
Fig. 3 Mean responses of adult female western flower thrips to different odors in a four-arm olfactometer. Time spent in the treated section was converted to a percentage of overall time, logit transformed, and compared to a test mean of -1.099 (logit transformation of $25 \%$ ) by onesample $t$-test. Data presented as $\pm 95 \%$ C.I., $* *_{-} P<0.01, *_{-}$ $P<0.05, N=12$

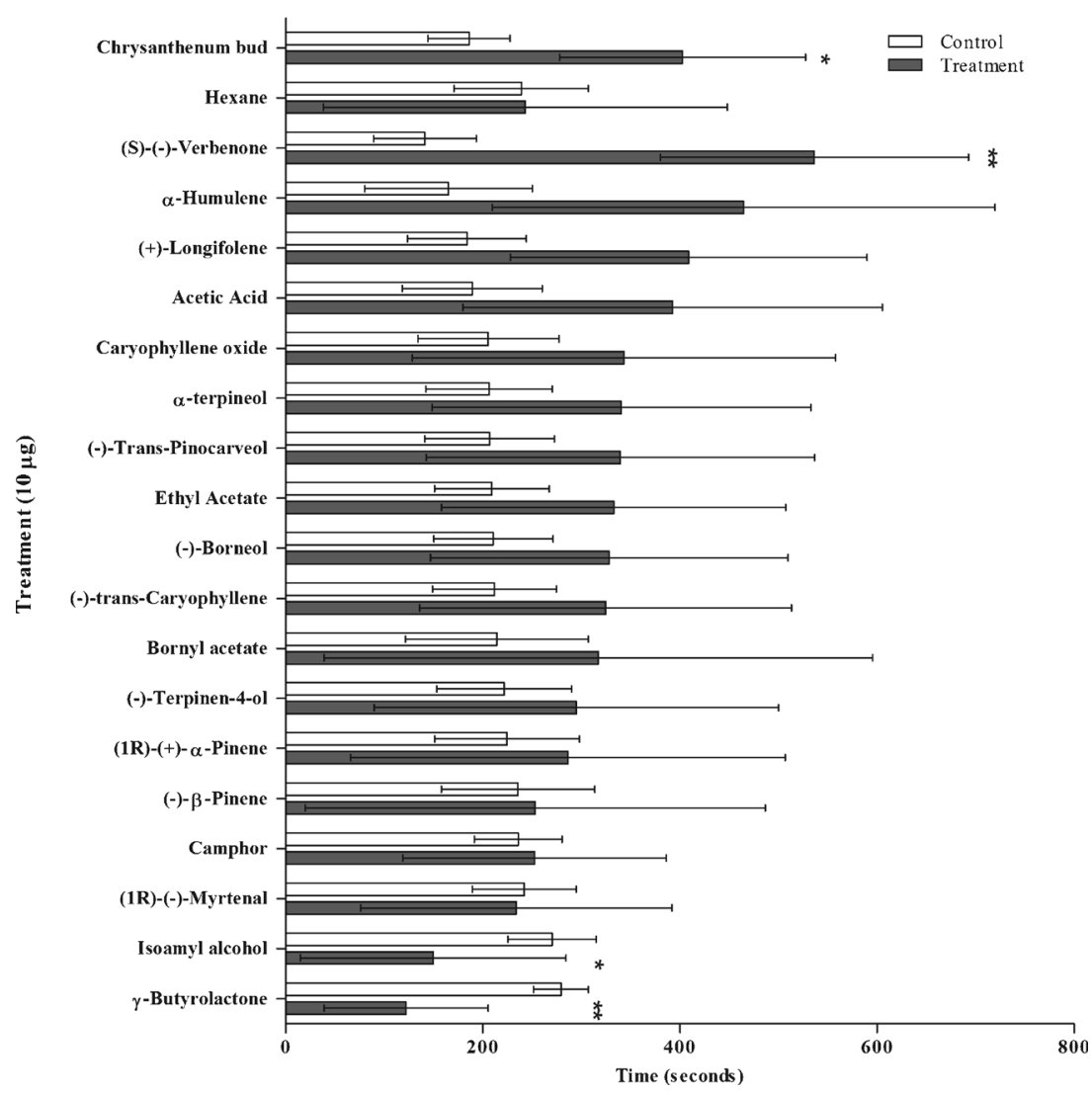

Thysanoptera may confirm whether a 'relict' olfactory receptor hypothesis is valid or not, as convergent evolution in many distinct species in a relatively short period of evolutionary time would be unlikely.

While most compounds identified in the headspace of pine pollen either elicited attraction $[(S)-(-)$-verbenone] or neutral responses from WFT, isoamyl alcohol and $\gamma$-butyrolactone appeared to repel WFT. These compounds are produced during anaerobic respiration by microorganisms and can often be detected during fermentation (Pietruszka et al. 2010; Vose et al. 2001). Thus, these compounds may be produced by microbes, rather than by the pollen. We speculate that WFT may utilize these compounds to determine the quality of nutrient sources; i.e., by avoiding food sources that are highly contaminated with microbes. The fact that WFT were attracted to pine pollen, even though these compounds were present, suggests that the ratio of attractants to repellents favored attraction. This is not unusual; many plants produce both attractant and repellent compounds, and it is the quantity and ratio or release rates that ultimately influence behavioral responses (Bruce et al. 2005). Alternatively, we may have tested these compounds at release rates much higher than that released naturally by pine pollen.

To summarize, we have shown that polyphagous WFT can detect and respond to volatiles from non-host species, which they may utilize as a supplementary nutrient source. Our research has identified compound(s) that could be used for monitoring or control of this important pest, and has demonstrated the value of researching non-primary hosts for novel semiochemicals.

Acknowledgments The European Union and Agrisense Ltd funded this work through Knowledge Economy Skills Scholarship (KESS). Western Flower Thrips were kindly provided by Dr. William Kirk of Keele University. We thank Dr. Ben Webster from the Department of Ecology, Swedish University of Agricultural Sciences for help with olfactometer construction and Dr. Christine Woodcock, Professor David Hall, and Dr. François Verheggen for assistance with EAGs. We are grateful for Richard Massy's assistance with olfactometer bioassays. We also thank Professor David Riley and the rest of the staff at the Department of Entomology in the University of Georgia for kindly providing the Pinus elliotti pollen sample used in this study.

Open Access This article is distributed under the terms of the Creative Commons Attribution License which permits any use, distribution, and reproduction in any medium, provided the original author(s) and the source are credited.

\section{References}

Anon (2009) Directive 2009/128/EC of the European Parliament and of 21 October 2009 establishing a framework for community action to achieve the sustainable use of pesticides. Off J Eur Comm L309:71 24.11.2009 
Bielza P (2008) Insecticide resistance management strategies against the western flower thrips, Frankliniella occidentalis. Pest Manag Sci 64:1131-1138

Bielza P, Quinoto V, Contreras J, Torne M, Martin A, Espinosa PJ (2007) Resistance to spinosad in the western flower thrips, Frankliniella occidentalis (Pergande), in greenhouses of south-eastern Spain. Pest Manag Sci 63:682-687

Bruce TJ, Wadhams LJ, Woodcock CM (2005) Insect host location: a volatile situation. Trends Plant Sci 10:269-274

Chitturi A, Riley DG, Joost PH (2006) Effect of pine pollen on settling behavior of Frankliniella occidentalis and Frankliniella fusca (Thysanoptera : Thripidae) on tomato and peanut. Environ Entomol 35:1396-1403

De Moraes CM, Mescheer MC, Tumlinson JH (2001) Caterpillar-induced nocturnal plant volatiles repel nonspecific females. Nature 410:577580

Dobson HEM (1987) Role of flower and pollen aromas in host-plant recognition by solitary bees. Oecologia 72:618-623

Dobson HEM, Bergstrom G (2000) The ecology and evolution of pollen odors. Plant Syst Evol 222:63-87

Friedman M, Henika PR, Mandrell RE (2002) Bactericidal activities of plant essential oils and some of their isolated constituents against Campylobacter jejuni, Escherichia coli, Listeria monocytogenes, and Salmonella enterica. J Food Prot 65:1545-1560

Gouinguené SP, Turlings TC (2002) The effects of abiotic factors on induced volatile emissions in corn plants. Plant Physiol 129:12961307

Grimaldi DA, Shmakov A, Fraser N (2004) Mesozoic thrips and early evolution of the order Thysanoptera (Insecta). J Paleontol 78:941952

Grotkopp E, Rejmanek M, Sanderson MJ, Rost TL (2004) Evolution of genome size in pines (Pinus) and its life-history correlates: Supertree analyses. Evolution 58:1705-1729

Hammer KA, Carson CF, Riley TV (2003) Antifungal activity of the components of Melaleuca alternifolia (tea tree) oil. J Appl Microbiol 95:853-860

Heming BS (1993) Structure, function, ontogeny, and evolution of feeding in thrips (Thysanoptera). In: Schaefer CS, Leschen RAB (eds) Functional morphology of insect feeding. Thomas Say Publications in Entomology. Entomological Society of America, Lanham, pp 3-41

Hulshof J, Vanninen I (2002) Western flower thrips feeding on pollen, and its implications for control. In: Marullo R, Mound L (eds) Thrips and Tospoviruses: Proc. 7th Intl. Symp. On Thysanoptera. Reggio Calabrio, Italy, pp 173-179

Kiritani K (2001) Invasive insect pests and plant quarantine in Japan. Extension Bulletin of the Food and Fertiliser Centre, Taipei 498:112

Kirk WDJ (1984) Pollen feeding in thrips (Insecta, Thysanoptera). J Zool Proc Zool Soc London 204:107-117

Kirk WDJ (1985) Pollen feeding and host specificity and fecundity of flower thrips (Thysanoptera). Ecol Entomol 10:281-289

Kirk WDJ (1987) How much pollen can thrips destroy? Ecol Entomol 12:31-40
Kirk WDJ (2002) The pest and vector from the West: Frankliniella occidentalis. In: Marullo R, Mound LA (eds) Thrips and tospoviruses: proceedings of the seventh international symposium on thysanoptera. Australian National Insect Collection, Canberra, pp 33-44

Kirk WDJ, Terry LI (2003) The spread of the western flower thrips Frankliniella occidentalis (Pergande). Agric For Entomol 5:301310

Labandeira CC (2006) The four phases of plant-arthropod associations in deep time. Geol Acta 4(4):409-438

Maddrell SHP (1969) Secretion by the Malpighian tubules of Rhodnius. The movement of ions and water. J Exp Biol 51:71-97

Morse JG, Hoddle MS (2006) Invasion biology of thrips. Ann Rev Entomol 51:67-89

Paine TD, Hanlon CC (1991) Response of Dendroctonus brevicomis and Ips paraconfusus (Coleoptera, Scolytidae) to combinations of synthetic pheromone attractants and inhibitors Verbenone and Ipsdienol. J Chem Ecol 17:2163-2176

Payne TL, Billings RF (1989) Evaluation of (S)-Verbenone applications for suppressing Southern Pine-Beetle (Coleoptera: Scolytidae) infestations. J Econ Entomol 82:1702-1708

Penalver E, Labandeira CC, Barron E, Delclos X, Nel P, Nel A, Tafforeau P, Soriano C (2012) Thrips pollination of Mesozoic gymnosperms. Proc Natl Acad Sci U S A 109:8623-8628

Pettersson J (1970) An aphid sex attractant. Entomol Scand 1:63-73

Pietruszka M, Przybylska KP, Szopa JS (2010) Synthesis of higher alcohols during alcoholic fermentation of rye mashes. Food Chem Biotechnol 74:51-64

Progar RA (2005) Five-year operational trial of Verbenone to deter Mountain Pine Beetle, Dendroctonus Ponderosae (Coleoptera: Scolytidae) attack of Lodgepole Pine (Pinus Contorta). Environ Entomol 34(6): 1402-1407

Riley DG, Chitturi A, Sparks AN Jr (2007) Does natural deposition of pine pollen affect the ovipositional behavior of Frankliniella occidentalis and Frankliniella fusca (Thysanoptera: Thripidae)? Ent Exp Appl 124:133-141

Riley DG, Angelella GM, McPherson RM (2011) Pine pollen dehiscence relative to thrips population dynamics. Ent Exp Appl 138:223-233

Schlyter F, Birgersson G, Leufven A (1989) Inhibition of attraction to aggregation pheromone by Verbenone and Ipsenol - density regulation mechanisms in Bark Beetle Ips Typographus. J Chem Ecol 15: 2263-2277

Shea PJ, Mcgregor MD, Daterman GE (1992) Aerial application of Verbenone reduces attack of Lodgepole Pine by Mountain PineBeetle. Can J For Res-Rev Can Rech For 22:436-441

Shore TL, Safranyik L, Lindgren BS (1992) The response of Mountain Pine-Beetle (Dendroctonus-Ponderosae) to Lodgepole Pine trees baited with Verbenone and Exo-Brevicomin. J Chem Ecol 18:533541

Tilden PE, Bedard WD (1988) Effect of Verbenone on response of Dendroctonus-Brevicomis (Coleoptera, Scolytidae) to ExoBrevicomin, Frontalin, And Myrcene. J Chem Ecol 14:113-122

Vose J, Tighe T, Schwartz M, Buel E (2001) Detection of gammabutyrolactone (GBL) as a natural component in wine. J Forensic Sci 46:1164-1167 